\title{
DEVELOPMENT OF A DATA ACQUISITION PROGRAM TO MEAS- URE THE EXHAUST TIME OF A PNEUMATIC VALVE
}

\author{
László Rónai \\ senior lecturer, Robert Bosch Department of Mechatronics, University of Miskolc \\ 3515 Miskolc, Miskolc-Egyetemváros, e-mail: ronai.laszlo@uni-miskolc.hu
}

\begin{abstract}
This paper deals with development of a data acquisition program to measure the exhaust time of a mechanical actuated pneumatic valve. The volume, which will be exhausted is determined by a so-called end of line testing procedure. The data acquisition unit is an Advantech ADAM 41178 channel analogue input module. The measuring and logging program is developed in LabVIEW software. The program provides the data in comma separated value file format for postprocessing opportunities.
\end{abstract}

Keywords: data acquisition, LabVIEW, pneumatic valve

\section{Introduction}

Nowadays measurement of physical parameters in manufacturing processes is essential to produce a quality product. These parameters can be, e.g., temperature, pressure, light, etc. Measurements can be the part of not only the manufacturing processes but also the testing operations. During a testing procedure the proper measurement of the quantities is important to make sure that the system can make stable decision about the correctness of the product. The development of sensors and data processing units has made it possible to easily integrate these elements into production.

Industry 4.0 (Rojko, 2017), (Lu, 2017) demands that all important parameters be measured and monitored during manufacturing, testing processes with minimal intervention. This approach makes it possible to increase productivity and make the traceability of manufactured products even more complete. In this case, the data of the measurements should be available in the future to ensure the opportunity of post-processing.

The main aim of this paper to develop a data acquisition program in LabVIEW software (Larsen, 2011), which can be used to measure the exhaust time of a concrete mechanically actuated pneumatic valve. The measurement is necessary to perform a testing process, which called end of line testing. This process has many segments, which is related to the pneumatic valve. In this paper the exhaust time of the valve between a supply pressure and a given pressure is determined.

Advantech ADAM 4117 module (Advantech, 2018) is used to collect the pressure values. This module has analogue voltage and current input capabilities. A Siemens SITRANS P 7MF4033 type pressure transmitter (Siemens, 2003) is used to measure the pressure.

This paper is organized as follows: Section 2 deals with the pneumatic valve and the definition of the measurement parameters and the basic description of the ADAM module. The configuration process of the data acquisition unit and the development of the measurement program is discussed in Section 3. Test measurements are performed to check the proper operation of the system. The concluding remarks will be given in Section 4 . 


\section{Description of the pneumatic valve and the measurement parameters}

A pneumatic valve can be used to drive and control pneumatic actuators for different purposes such as industrial tasks or home applications. There are several types of valves, and it can be characterized by its numbers of connection ports and states.

Our task contains an Aventics 3/3-way pneumatic valve, which has 3 connection ports and 3 states. The symbol of the valve is shown in Figure 1. The mechanical actuation is performed by a stem. In the rest position of the valve the flow rate setting from work port 2 to ventilation port 3 is predefined to act upon the exhaust time. The main goal is to measure the exhaust time in that position depending on the test conditions.

Before testing the valve, some parameters must be defined: the volume after the work port is needed to be $36,4 \mathrm{ml}$, the supply pressure $p_{1}$ should be 9.3 bar. The exhaust time needs to be within the range $10-15 \mathrm{sec}$. The time is determined from the release of the pressurized state of work port 2 to the middle state of the valve till the pressure reaches an exact value, which is 4.9 bar.

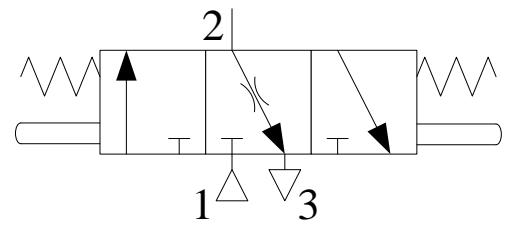

Figure 1. Symbol of the mechanically actuated pneumatic valve

The volume after port 2 will be provided by application of a pneumatic tube to ensure the test condition. To measure the pressure a transmitter and a data acquisition unit are required. A Siemens brand pressure transmitter and an Advantech ADAM 4117-AE 16-bit resolution data acquisition module are used. The transmitter is adjusted to provide 4-20 mA between the range of 0-10 bar input pressure. The channel 0 of the ADAM module is used to measure the output current of the transmitter. Therefore, this input port is set to measure current values. The module has eight analogue input capability, which have programmable input ranges (Advantech, 2018). The unit has many supported input ranges for unipolar and bipolar purposes, e.g., 0-10 V, 0-1 V, 0-5 V, 0-20 mA, 4-20 mA, $\pm 1 \mathrm{~V}, \pm 10 \mathrm{~V}, \pm 20 \mathrm{~mA}$ etc.

The RS-485 pins A and B of the acquisition unit is connected to a RS-485-USB converter module to read the raw measurement values with a personal computer. Advantech Adam Apax .NET Utility software can be used to configure the unit. Before configuration, the ADAM module should be in INIT mode, which is provided by a switch located on the unit. The baud rate is set to $38400 \mathrm{bps}$, and the Advantech protocol is used. Therefore, the data format is the following: 1 start bit, 8 data bits, and 1 stop bit with no parity (Advantech, 2018). The ID of the unit will be $01 \mathrm{~h}$. After the configuration process, the unit is switched to normal mode.

The module needs commands from the PC to make configurations and to query the measured data. The whole command syntax can be found in (Advantech, 2018). These syntaxes will be used to develop an acquisition program in LabVIEW. The configuration of single channel, read single channel type/range, and read from single analogue input commands will be used. The beginning of every command contains a delimiter character, e.g., \#, \$, \%. When developing the program, special attention had to be paid to ensure easy parameterization. 


\section{The measuring program}

The flow chart of the developed program is shown in Figure 2. The program can be divided to three independent parts. The main part consists of the data measuring and storing capabilities, and the other two parts contains the configuration of the selected channel and the reading of the settings of the selected single channel.

The user can give the communication port (COM port), the number of the measuring channel, the baud rate, the ADAM ID, the input type, and the range of the analogue input through a graphical user interface (GUI), which can be seen in Figure 3. The ADAM ID is a two-character hex address, which identify the concrete module.

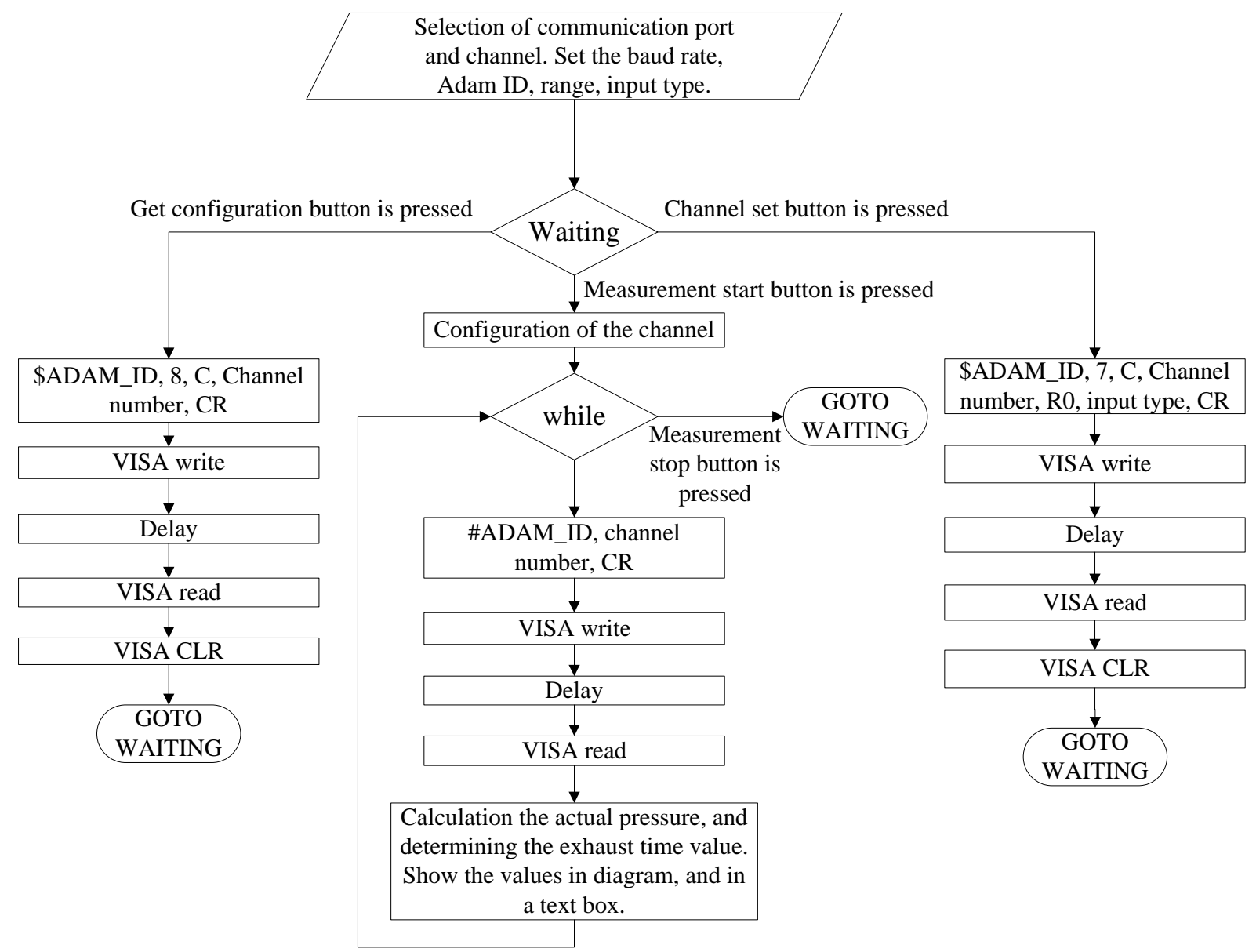

Figure 2. The flow chart of the program

The main part of the program will be active when the Measurement start button is pressed until Measurement stop button is not actuated. Thereafter the initial values of the variables, e.g., exhaust time is set, and the serial port is opened. A Flat sequence structure provides the sequential process of the program. This sequence is placed in a while loop. The first step is to create the command, i.e., \#010 with carriage return (CR) in our case to the ADAM module to provide the measurement values of the channel 
0 with ADAM ID 01h. Carriage return is the terminating character in the commands. The second step contains the command sending part with VISA Write function. A delay is placed in another frame of the Flat sequence structure to set the sampling time. During the measurements, the sampling rate is set to $50 \mathrm{~Hz}$. Thereafter in the next frame the reading of the raw value is produced by the VISA Read function.

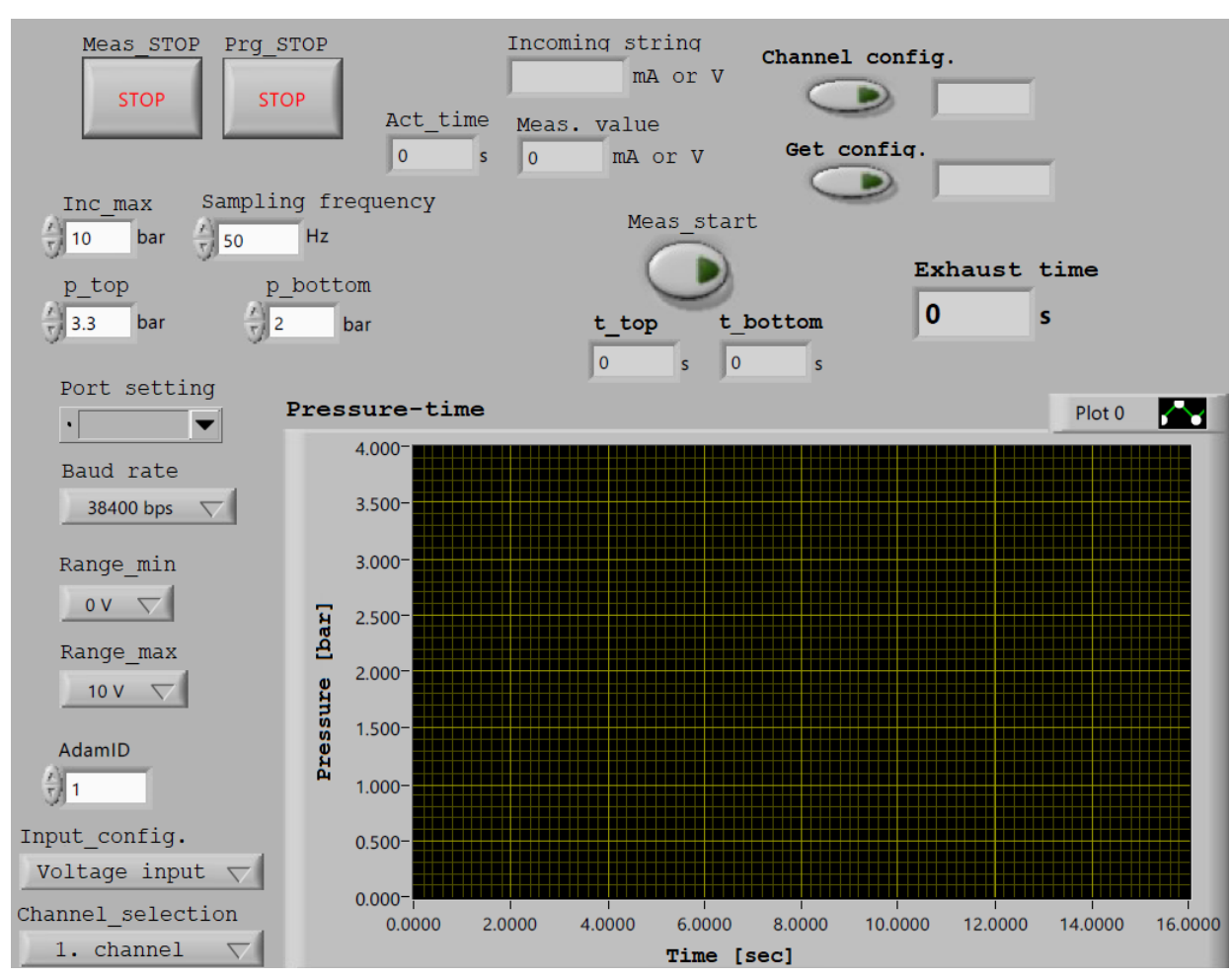

Figure 3. GUI in LabVIEW

Due to 4-20 mA incoming signal, the raw value should be transformed to pressure value:

$$
p_{\text {measured }}=\frac{I_{\text {incoming }}-4 m A}{20 m A-4 m A} p_{\text {max }}
$$

where $I_{\text {incoming }}$ is the incoming raw current value, $p_{\max }$ is the maximum pressure, which can be occurred during the measurements. The block Build XY Graph is capable to produce the plot of the measurement, with pressure versus time. This part of the program can determine the exhaust time value depending on the pressure values:

$$
t_{\text {exhaust }}=t_{\text {bottom }}-t_{\text {top }}
$$

where $t_{\text {bottom }}$ is the time stamp, where the pressure reaches the 4.9 bar limit value, and $t_{\text {top }}$ is the last time stamp, where the pressure was around 9.3 bar. These time values are ensured by comparison blocks.

In order to read the configurational settings of an exact channel before measurements, a program block was developed (see Figure 4.). Get configuration data button needs to be pressed to start this process. The command is assembled by concatenating input strings, which are the delimiter \$, ADAM ID, characters 8 and $\mathrm{C}$, and the number of the channel. In our case the command is the following \$018C0 with CR. After assembling the string, the program is sending to the ADAM module. The response of 
the module contains the type and the range of the channel, e.g., !01C0R07 with CR. After delimiter ! the first two character are the ADAM ID it is followed by the number of the channel, R07 means, that the channel is configured to measure current within the range 4-20 mA.

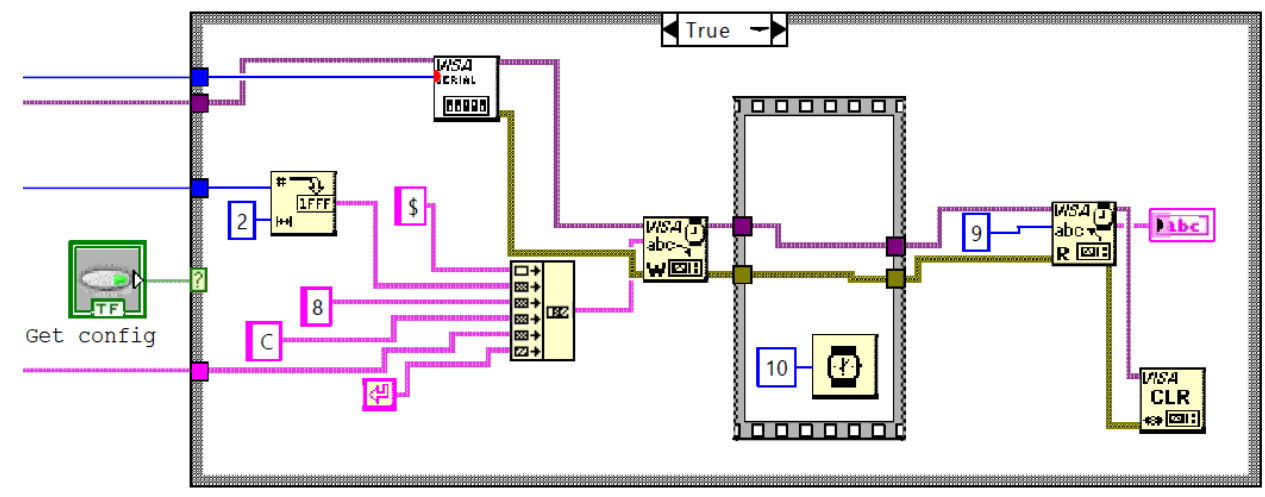

Figure 4. Program block to read configurational settings of the selected single channel.

The configuration program of a single channel is shown in Figure 5. When the configurational settings of an exact channel are not appropriate, Channel set button can be pressed to perform the configurational operation. Command string is concatenated, e.g., \$017C0R07 with CR, where R07 means that the channel is configured to measure current between 4-20 mA, and after the ADAM ID, number 7 refers to the configuration process. With VISA Write function the command is sent to the selected module. Thereafter the program can read the answer of the module with the use of VISA Read function.

The response of the module is the following during a proper operation: !01 with CR. The delimiter ! means that a valid command was received and $01 \mathrm{~h}$ is the ADAM ID. Therefore, in this case the module is configured successfully.

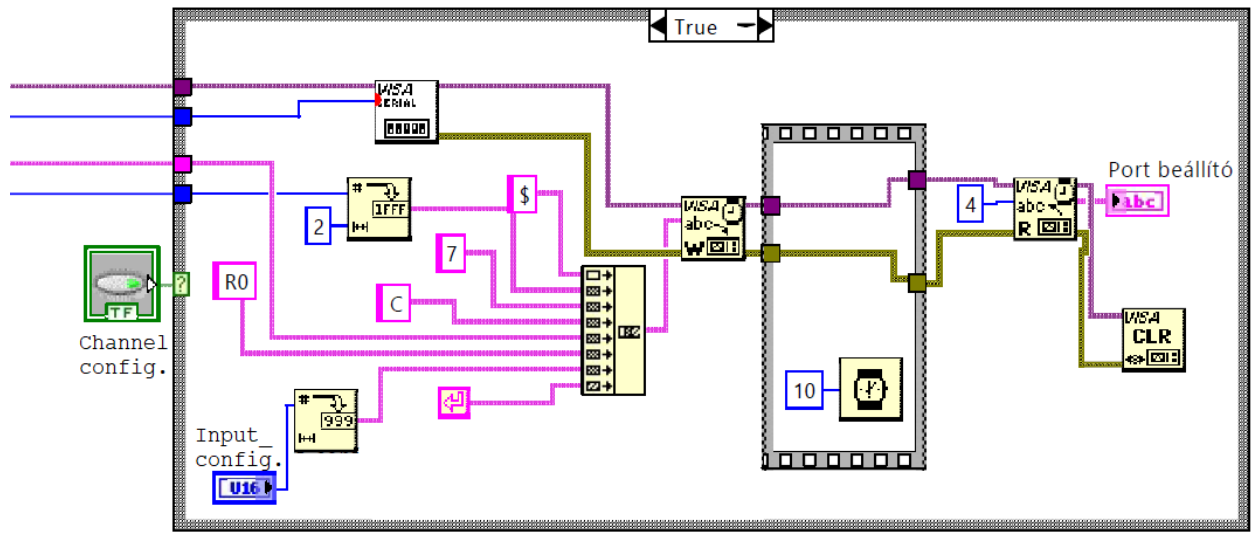

Figure 5. Block to configure the selected channel.

One of the measurements is shown in Figure 6. The measurement data can be saved in .csv file format to create diagrams. At the vicinity of time $3 \mathrm{sec}$, the work port 2 of the pneumatic valve is pressurized. Some seconds later the valve is switched to its middle state. As we can see the pressure is decreasing continuously due to the choke element. When the 4.9 bar limit is reached the program can calculate the exhaust time of the valve. In our case it is $13.5 \mathrm{~s}$. 


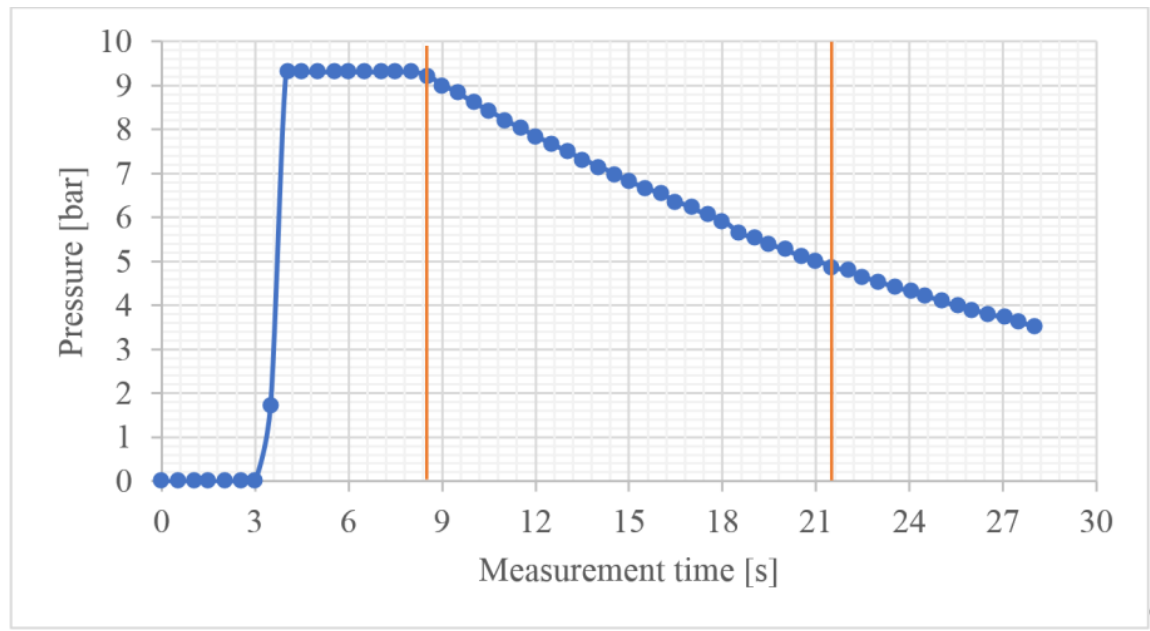

Figure 6. Measurement results

The developed program is well applicable to determine the exhaust time of pneumatic valves.

\section{Summary}

The development of a data acquisition program in LabVIEW software is discussed. The program is capable to use in different industrial measurements with an ADAM 4117 data acquisition module. In this paper the exhaust time of a mechanically actuated pneumatic valve was determined. The calculation of this time parameter is the part of the so-called end of line testing process to ensure the proper quality of the product.

The measurements showed that the developed program is well applicable with the ADAM data acquisition module to collect and evaluate measurement data.

\section{Acknowledgements}

The research reported here was carried out as part of the EFOP-3.6.1-16-2016-00011 "Younger and Renewing University - Innovative Knowledge City - Institutional development of the University of Miskolc aiming at intelligent specialization" project implemented in the framework of the Szechenyi 2020 program. The realization of this project is supported by the European Union, co-financed by the European Social Fund.

\section{References}

[1] Rojko, A. (2017). Industry 4.0 Concept: Background and Overview. Int. J. of Interact. Mobile Techn., 11(5), 77-90. https://doi.org/10.3991/ijim.v11i5.7072

[2] Lu, Y. (2017). Industry 4.0: A Survey on Technologies, Applications and Open Research Issues. J. Ind. Inf. Integr., 6, 1-10. https://doi.org/10.1016/j.jii.2017.04.005

[3] Larsen, R. W. (2011). LabVIEW for Engineers. Prentice Hall, Upper Saddle River, New Jersey.

[4] Advantech Co., Ltd.: User Manual: ADAM-4100 Series, $2^{\text {nd }}$ edition, Part No. 2003M41001, 2018

[5] Siemens AG: Instruction Manual SITRANS P, DS III series 7MF4*33-, A5E00047092-05, Siemens AG, D-76181 Karlsruhe, 2003 